\title{
ADHESION AND FRICTION FORCE MEASUREMENTS IN AMBIENT AND HIGH VACUUM CONDITIONS
}

\author{
M.A. YAQOOB ${ }^{1,2}$, M.B. DE ROOIJ ${ }^{2} \&$ D.J. SCHIPPER ${ }^{2}$ \\ ${ }^{1}$ Materials Innovation Institute, Delft, The Netherlands. \\ ${ }^{2}$ Laboratory of Surface Technology and Tribology, University of Twente, Enschede, The Netherlands.
}

\begin{abstract}
Physical insight into the frictional behaviour of surfaces in contact with vacuum and other special environments is important for the accuracy of positioning mechanisms operating in these environments. The positioning accuracy and drift in these mechanisms are strongly influenced by the frictional behaviour of the mating materials. The cause for both drift and positioning accuracy is stick-to-slip and slip-to-stick transitions at asperity level, resulting in a displacement at macrolevel. Adhesion as well as friction experiments were performed for single asperity and multi-asperity contacts both in ambient and high vacuum conditions on a novel designed vacuum-based adhesion and friction tester. This paper discusses the experimental setup designed and manufactured to investigate the adhesion and friction behaviour of a single asperity contact. The intrinsic roughness of the ball and the flat will form a multiasperity contact. Pull-off and friction force measurements can be performed with the resolution better than $5 \mu \mathrm{N}$. The maximum normal load that can be applied with this system is $100 \mathrm{mN}$. The setup is capable of working at $10^{-6}$ mbar vacuum level as well as in ambient conditions. Experimental results show that the surrounding environment and roughness play an important role both in the adhesion force and friction force measurements. The friction force measurements show good agreement with the basic theories of contact mechanics.

Keywords: Adhesion force, force-displacement curve, friction force, high vacuum, mechanical vibrations, multi-asperity contact, positioning accuracy, single asperity contact
\end{abstract}

\section{INTRODUCTION}

Adhesion force is present between two surfaces when brought closer or made contact with each other. This force is generally caused by the superposition (expressed in eqn (1), [1]) of different kinds of surface forces like van der Waals forces, electrostatic forces, capillary forces and other interacting surface forces, see Israelachivili [2]

$$
F_{a}=F_{v d w}+F_{c a p}+F_{e l}+\ldots
$$

where $F_{a}(N)$ is the adhesion force, $F_{v d w}(N)$ is the van der Waals force, $F_{c a p}(N)$ is the capillary force and $F_{e l}(N)$ is the electrostatic force. The adhesion force strongly depends on many physical factors such as surface energy, surface roughness, geometry and size, separation, applied normal load, environmental conditions like pressure and temperature, duration of contact, hydrophilic or hydrophobic nature of the contacting surfaces, see Bhushan [3].

Water is present on any (hydrophilic) surface in an ambient (humid) environment. The water layers are present on the surface and the thickness of this layer depends on the relative humidity, see Israelachivili [2]. When two surfaces come in close proximity or contact the water layers form a meniscus and the surface will stick together. Capillary/meniscus force, if present, are dominant and contribute most to the total adhesion force present in the contact as explained by van Zwol et al. [4]. However, if the surfaces are rough this adhesion force due to capillary formation will reduce two orders of magnitude with one order of rms roughness changes, see van Zwol et al. [4]. 
The influence of adhesion on the contact was investigated by developing different theories and models at macro, micro and nanoscale, see Johnson and Greenwood [5]. In case of micro and nanoscale experiments, the fundamental laws of friction are not obeyed and the system is dominated by adhesion-influenced contact mechanics, see Feldman et al. [6]. Johnson, Kendall and Roberts (JKR), Derjaguin, Muller and Topolov (DMT), Tabor and MaugisDugdale (MD) presented contact mechanics models for elastic deformation in the contact incorporating the adhesion force. Furthermore, the work of adhesion in the contact can be calculated by measuring the adhesion force or pull-off force and using the applicable contact model for the system, see Johnson and Greenwood [5].

The adhesion and friction forces are present when the surfaces are in contact under an applied normal load and are subjected to a lateral (tangential) motion. The friction force can be divided into two regimes, the static friction regime and the dynamic friction regime. Before sliding occurs, so during the static friction regime, there is always a displacement in the order of nanometres present when a tangential load is applied to move the two surfaces relative to each other in lateral direction [7-9]. This displacement is termed as preliminary displacement or micro-slip. The presence of this preliminary displacement causes positioning errors at start/stop positions.

Friction, as related to positioning accuracy, is often studied for control purposes. To develop more accurate control algorithms, friction is taken as a part of the dynamical system; thus, it makes the control algorithms complicated and nonlinear in nature. An extensive number of models have been developed for this purpose as explained by Amstrong-H'elouvry [10] and Capone et al. [11]. Such models are typically aimed at modelling effects like a velocity dependency of dynamic friction.

In this paper, the experimental setup designed and manufactured for performing adhesion and friction measurements at microlevel in different environments like ambient air, dry nitrogen atmosphere and high vacuum is discussed. Measurements performed both in ambient air and vacuum for different material combinations will be discussed and the results will be compared with the existing theory.

\section{DESIGN}

A complete new vacuum-based test rig was designed, manufactured, assembled and tested. The main aim of this setup is to perform adhesion and friction measurements at microscale in ambient $\left(20^{\circ} \mathrm{C}, 1\right.$ bar and $50 \%$ relative humidity) as well as in special environments like high vacuum $\left(20^{\circ} \mathrm{C}\right.$ and $\left.10^{-6} \mathrm{mbar}\right)$ and dry nitrogen. This vacuum adhesion and friction tester (VAFT), as shown in Fig. 1, comprises three positioning stages and two capacitive sensors along with a force measuring mechanism as shown in Fig. 1c. The setup has a ball on flat configuration and represents a single asperity contact. The ball is mounted on the indenter and the indenter along with the force measuring mechanism is mounted on one of the positioning stages, which can move in $\mathrm{Z}$ direction as shown in Fig. 1c. This positioning stage is used to make contact with the flat surface and to apply the normal load. The flat surface is placed on an XY stage. The X positioning stage is used to apply a tangential load for friction measurements. The accuracy of both $\mathrm{X}$ and $\mathrm{Z}$ stage is $20 \mathrm{~nm}$ with a stroke of $20 \mathrm{~mm}$. The $\mathrm{Y}$ stage is used to perform multiple parallel measurements on the flat surface and has a stroke of $20 \mathrm{~mm}$ as well.

The measuring range of the capacitive sensors is $50 \mu \mathrm{m}$ with an accuracy better than $1 \mathrm{~nm}$. The capacitive sensors are mounted on the force measuring mechanism. The stiffness of the force measuring mechanism is calibrated. By measuring the deflection of this mechanism with the help of capacitive sensors, the force can be calculated, see Yaqoob et al. [12]. 

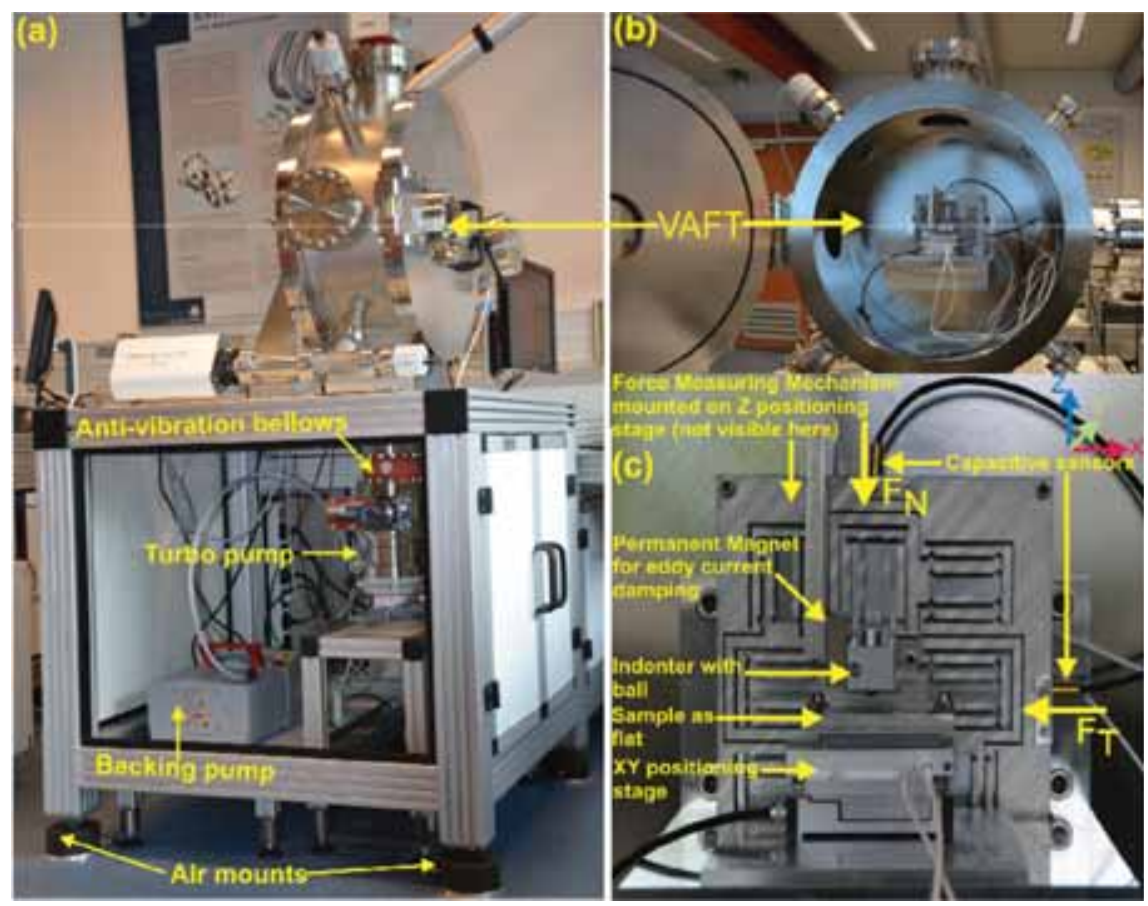

Figure 1: (a) Description of components mounted to reduce vibrations. (b) Adhesion and friction tester mounted inside the vacuum chamber. (c) Internal view of VAFT showing all the components.

Mechanical disturbances are the major potential sources of instability and inaccuracy in a system. In this system, these disturbances are mainly categorized as ground vibrations, vibrations from the vacuum pump and vibrations induced due to dynamic effects of the moving $\mathrm{Z}$ positioning stage. The complete system is mounted inside a vacuum chamber as shown in Fig. 1b. A special three-step damping technique was used to reduce these disturbances as explained by Yaqoob et al. [12].

The heart of the VAFT is the force measuring mechanism. The mechanism is designed to have two DOFs for measuring the normal and the friction force. The conceptual design of this mechanism has been explained by Awtar [13]. It was required to have a mechanism which can measure the two perpendicular forces independently. The mechanism consists of eight compound parallelogram frictionless hole-hinge flexure mechanisms as shown in Fig. 2.

In this mechanism, there are four rigid stages: ground, motion stage and two intermediate stages as shown in Fig. 2. The intermediate stages are necessary to decouple and isolate the motion of the two axes. The four compliant units are called Flexure A, B, C and D and their respective mirrored compliant units are Flexure $A^{\prime}, B^{\prime}, C^{\prime}$ and $D^{\prime}$. When the normal force is applied the Flexure B, B' and D, $\mathrm{D}^{\prime}$ would bend to give the desired displacement and Flexure $\mathrm{A}, \mathrm{A}^{\prime}$ and $\mathrm{C}, \mathrm{C}^{\prime}$ are in tensile/compressive load. Similarly, when the lateral force is applied flexure $\mathrm{A}, \mathrm{A}^{\prime}$ and $\mathrm{C}, \mathrm{C}^{\prime}$ deflects to give the desired motion and Flexure $\mathrm{B}, \mathrm{B}^{\prime}$ and $\mathrm{D}, \mathrm{D}^{\prime}$ are in tensile/compressive load. Any parasitic errors due to bending of compound flexures are compensated by the secondary motion stage. Furthermore, this force measuring mechanism is relatively insensitive to thermal disturbances and manufacturing errors due to its symmetry. 


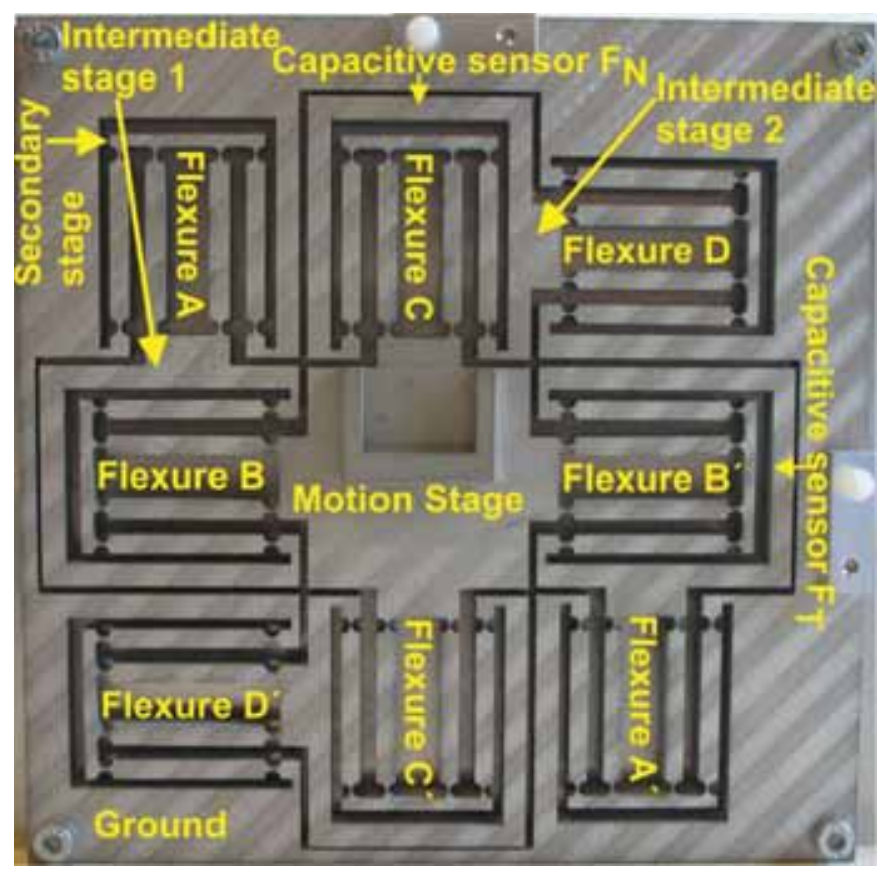

Figure 2: Detailed design of the force measuring mechanism showing the primary and secondary moving stages and the compound parallelogram hole-hinge flexure mechanism.

The force measuring mechanism has been calibrated and the stiffness of the mechanism has been calculated. The calibrated stiffness of the force measuring mechanism calculated by the slope of the force-displacement curve is $3.75 \mathrm{mN} / \mu \mathrm{m}$, see Yaqoob et al. [12].

One of the challenges in developing this setup was to build a routine for finding a reference position accurately where the ball and the flat are in contact. As explained earlier that the system has intrinsic vibrations which were reduced as much as possible, these vibrations were used to find the contact. When the ball and the flat are not in contact the capacitive sensors will read the deflection of the hinges which will be varying with the resonance frequency of the hinges. The resonance frequency of the hinges is $30 \mathrm{~Hz}$ and as soon as the ball touches the flat surface the resonance frequency of the system shifts to $80 \mathrm{~Hz}$ due to the increase in the stiffness of the system. The power spectrum of the deflection signal from the sensors is used to find this frequency shift and used as an input in the control software for controlling the stages developed in LabView.

\section{RESULTS AND DISCUSSION}

Experiments were performed with two kinds of contacting bodies. The first type depicts a single asperity contact with silicon $(\mathrm{Si})$ and silica $\left(\mathrm{SiO}_{2}\right)$ ball and a smooth float glass flat surface, referred as $\mathrm{Si}-$ Glass and $\mathrm{SiO}_{2}-$ Glass interface. The second type is a relatively rough sapphire $\left(\mathrm{Al}_{2} \mathrm{O}_{3}\right)$ ball and a smooth float glass flat, $\mathrm{Al}_{2} \mathrm{O}_{3}$-Glass interface and Zirconia $\left(\mathrm{ZrO}_{2}\right)$ ball and a rough $\mathrm{ZrO}_{2}$ flat surface, referred as $\mathrm{ZrO}_{2}-\mathrm{ZrO}_{2}$ interface, representing a multi-asperity contact. These materials were used to perform adhesion experiments both in ambient and high vacuum conditions. The friction experiments were performed only with 
Table 1: Material properties of the contacting surfaces.

\begin{tabular}{|c|c|c|c|c|}
\hline Material Properties & $\begin{array}{l}\text { Elastic modulus } \\
\qquad E(\mathrm{GPa})\end{array}$ & $\begin{array}{c}\text { Surface energy, } \\
\quad \gamma\left(\mathrm{mJ} / \mathrm{m}^{2}\right)\end{array}$ & $\begin{array}{c}\text { Roughness, } \\
R_{q}(\mathrm{~nm})\end{array}$ & $\begin{array}{l}\text { Radius, } \\
R \text { (mm) }\end{array}$ \\
\hline Si ball & 168 & $44.1 \pm 3.1^{*}$ & 2 & 2.5 \\
\hline $\mathrm{SiO}_{2}$ ball & 74 & $44.1 \pm 3.1$ & $3-5$ & 2.5 \\
\hline Sapphire ball & 463 & 41.1 & $8-10$ & 2.5 \\
\hline Float Glass flat & 74 & 83.4 & 1 & $\infty$ \\
\hline $\mathrm{ZrO}_{2}$ ball & 200 & 45.6 & 550 (Fig. 4) & 0.4 \\
\hline $\mathrm{ZrO}_{2}$ flat & 200 & 45.6 & 500 (Fig. 4) & $\infty$ \\
\hline
\end{tabular}

*Surface energy of $\mathrm{SiO}_{2}$ because of oxide layers present on the surface.

$\mathrm{Si}-$ Glass interface both in ambient and high vacuum (HV) conditions at different applied normal loads. The material properties of the materials used are shown in Table 1 and were reported by Xu et al. [14], Harnett et al. [15] and Krol and Krol [16].

\subsection{Adhesion force measurements}

Adhesion force measurements were performed with $\mathrm{Si}-\mathrm{Glass}, \mathrm{SiO}_{2}-\mathrm{Glass}, \mathrm{Al}_{2} \mathrm{O}_{3}-\mathrm{Glass}$ and the $\mathrm{ZrO}_{2}-\mathrm{ZrO}_{2}$ combinations both in ambient and vacuum. A typical force-displace ment curve for the Si-Glass combination in ambient is shown in Fig. 3. This forcedisplacement curve represents the applied normal load plotted against the vertical displacement of the $\mathrm{Z}$ stage. In Fig. 3, the three different areas of the force-displacement curve are shown. Two distinctive regions of the force-displacement curve are shown which represents before and after the contact situation. The $\mathrm{Z}$ stage starts moving downward from the home position, until the desired normal load is applied after making the contact. This section of the curve is called "Loading" or approach and is represented by the dashed line. The part of the measurement where the contact is made and the flat surface is loaded and unloaded is also shown in the insets of Fig 3. The hysteresis in the loading-unloading loop is typical adhesion hysteresis because the work needed to separate two surfaces is greater than the work that was gained by bringing them together as explained by Yoshizawa et al. [17] and Liu and Bushan [18].

The exact pull-off point is shown in the inset of Fig. 3a, which is zoomed in area where the two surfaces are brought in contact. The contact is broken from the surface at the "Pull-off point" when the Z stage is moving upward as shown in the inset of Fig. 3a. This section of the curve is called "unloading" or retract and is represented by the solid line. The negative value of the force represents the adhesion force present in this particular system and environment. Furthermore, it can be seen from the graphs that the flat surface has been loaded with $10 \mathrm{mN}$ by the silicon ball. The corresponding adhesion force is about $1 \mathrm{mN}$. After the breakaway the dynamic effects in the measurement data can be seen.

With the same system, the adhesion force measurements in vacuum have also been performed and similar force-displacement curves at a pressure of $10^{-6}$ mbar is shown in Fig 3b. A significant difference in the pull-off force is observed when measured in vacuum compared with ambient. With the applied normal load of $10 \mathrm{mN}$, the adhesion force measured is about $380 \mathrm{mN}$. 

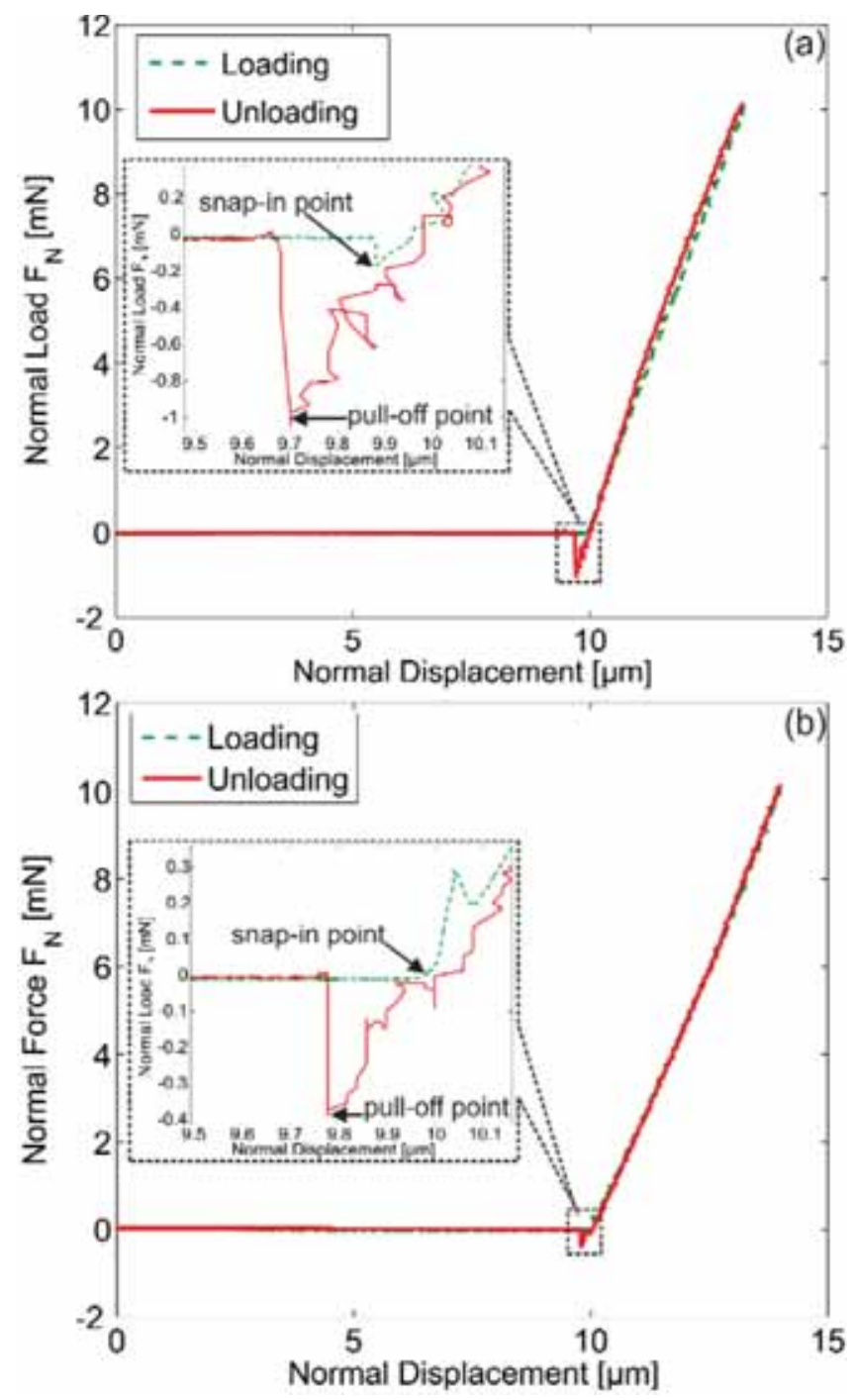

Figure 3: A typical force-displacement curve between vertical displacement and normal load to measure adhesion force in (a) ambient and in (b) HV conditions.

The contact time for both measurements was kept the same to eliminate any transient effects. This significant difference in the pull-off force in vacuum conditions is because of the different magnitude of the meniscus and van der Waals forces that are contributing to the adhesion force [19]. It is evident from this result that the Si-Glass interface undergoes a completely different contact situation only because of the change in the surrounding environment.

Adhesion force measurements both in $\mathrm{HV}$ and ambient were also performed with a rough $\mathrm{ZrO}_{2}$ ball against a rough $\mathrm{ZrO}_{2}$ flat surface. The height profile measurements performed with the Keyence Laser Scanning Microscope are shown in Fig. 4. The scan area is $145 \times 109 \mu \mathrm{m}^{2}$ for both the spherical and flat surfaces. The roughness measurement of the ball is performed 


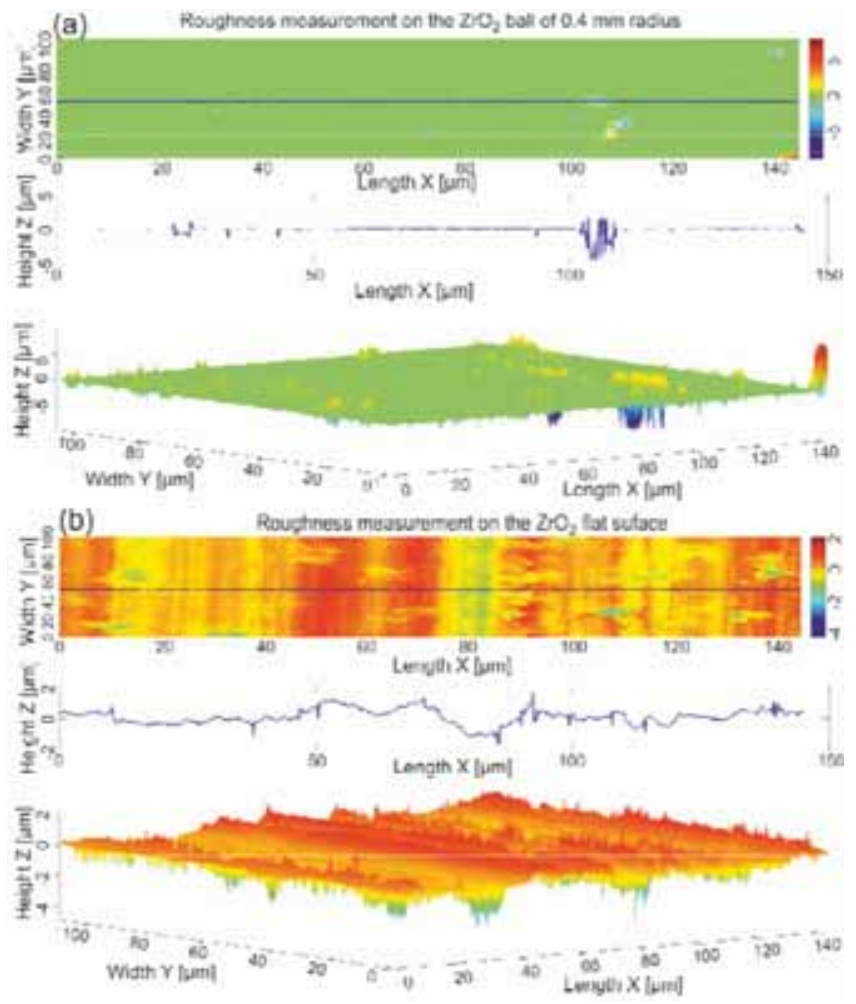

Figure 4: (a) Roughness measurement of $0.8-\mathrm{mm}_{\mathrm{ZrO}_{2}}$ ball. The figure shows the height profile, line profile and a 3D image of the flattened ball. (b) Roughness measurement of $\mathrm{ZrO}_{2}$ flat surface.

after correcting the tilt and spherical profile into a plane. The corresponding rms line roughness values for $\mathrm{ZrO}_{2}$ ball and $\mathrm{ZrO}_{2}$ flat surface are 550 and $500 \mathrm{~nm}$, respectively. The pull-off force for the multi-asperity contact in both environments was significantly lower than the single asperity contact. There are small microcontacts present in the contact and, therefore, the real area of contact is much smaller than the nominal area of contact, which reduces the pull-off force significantly. Furthermore, capillary force and the van der Waals force scales with the radius of the spherical surface (here contacting asperities), see Israelachivili [2]. Similar results have been reported by van Zwol et al. [4]. Rabinovich et al. [20] and van Zwol et al. [4] also showed that the adhesion force reduces two orders of magnitude with the increase of rms roughness from 1 to $10 \mathrm{~nm}$.

In Fig. 5, the results are shown for the adhesion measurements of the $\mathrm{ZrO}_{2}-\mathrm{ZrO}_{2}$ interface. The normal load of $10 \mathrm{mN}$ is applied on the $\mathrm{ZrO}_{2}$ flat surface with a $\mathrm{ZrO}_{2}$ ball of diameter of $800 \mu \mathrm{m}$. Furthermore, when the system is unloaded a force of about $30 \mathrm{mN}$ is required to break free the contact as shown in the inset of Fig. 5a. This force is significantly lower than the pull-off force as shown in Fig. 3. There are many reasons for a lower pull-off force for $\mathrm{ZrO}_{2}-\mathrm{ZrO}_{2}$ interface. First, the radius of the $\mathrm{ZrO}_{2}$ ball is much smaller than the $\mathrm{Si}$ ball and the adhesion force scales with the radius of the sphere. Secondly, the roughness values of the $\mathrm{ZrO}_{2}-\mathrm{ZrO}_{2}$ interface and the $\mathrm{Si}-$ Glass interface are very different. The adhesion force decreases significantly for the rough surface as compared with smooth surfaces, 


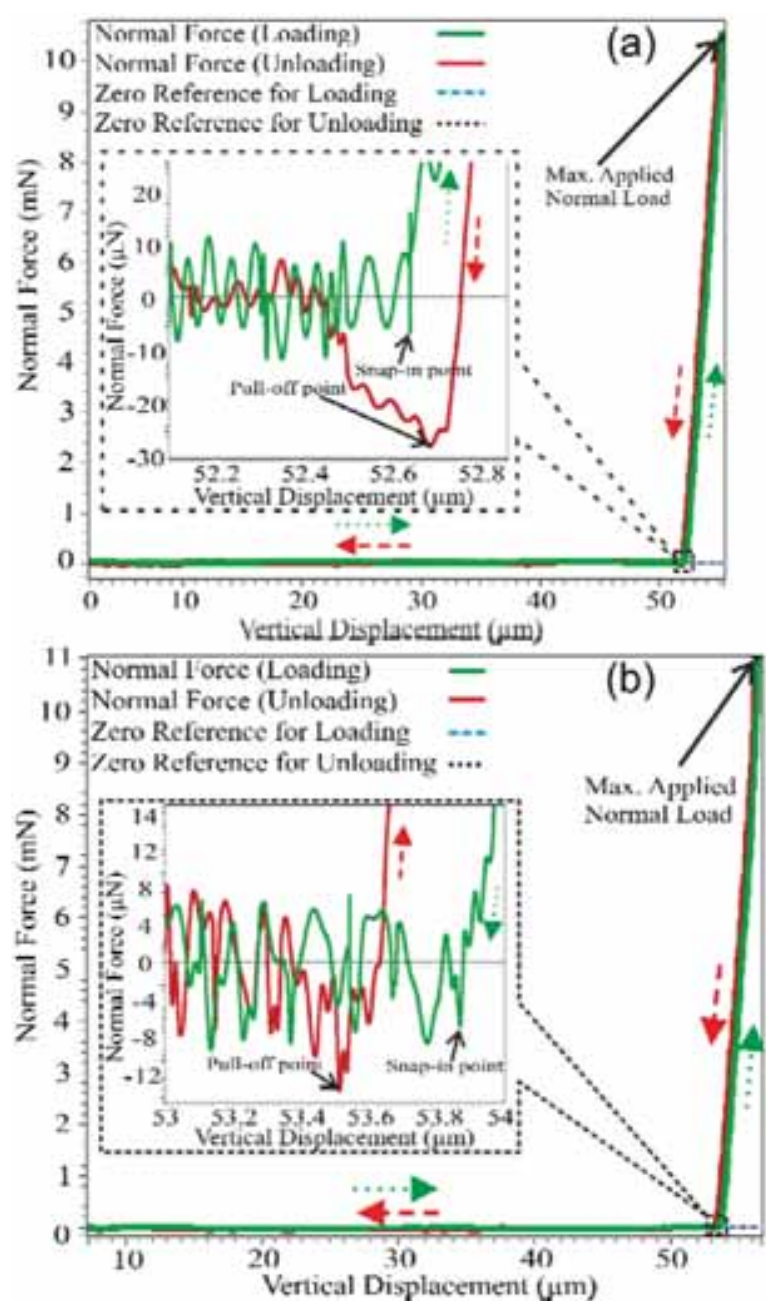

Figure 5: Adhesion force measurement between $\mathrm{ZrO}_{2}$ ball and $\mathrm{ZrO}_{2}$ flat surface in (a) ambient and (b) HV conditions.

see van Zwol et al. [4]. Thirdly, the work of adhesion or surface energy also influences the adhesion force which is not of that importance in ambient conditions since the adhesion force is dominated by the capillary force. Also, as can be seen in Table 1 the surface energy of $\mathrm{SiO}_{2}$ and $\mathrm{ZrO}_{2}$ is very close to each other, so this might not influence significantly the adhesion force. However, the hydrophilicity of the surfaces is important since the capillary force strongly depends on the contact angle of the surface.

Similarly, the adhesion force for the $\mathrm{ZrO}_{2}-\mathrm{ZrO}_{2}$ interface in $\mathrm{HV}$ conditions is measured. The applied normal load in this case is $11 \mathrm{mN}$ and the corresponding pull-off force is approximately $13 \mu \mathrm{N}$. So it can be deduced that the pull-off force is reduced 50\% when operated in vacuum for multi-asperity contact. This decrease is somewhat different than the reduction in pull-off force for the single asperity contact which was about $60 \%$. However, the magnitude of adhesion force in $\mathrm{HV}$ for single and multi-asperity contact is significantly different. 


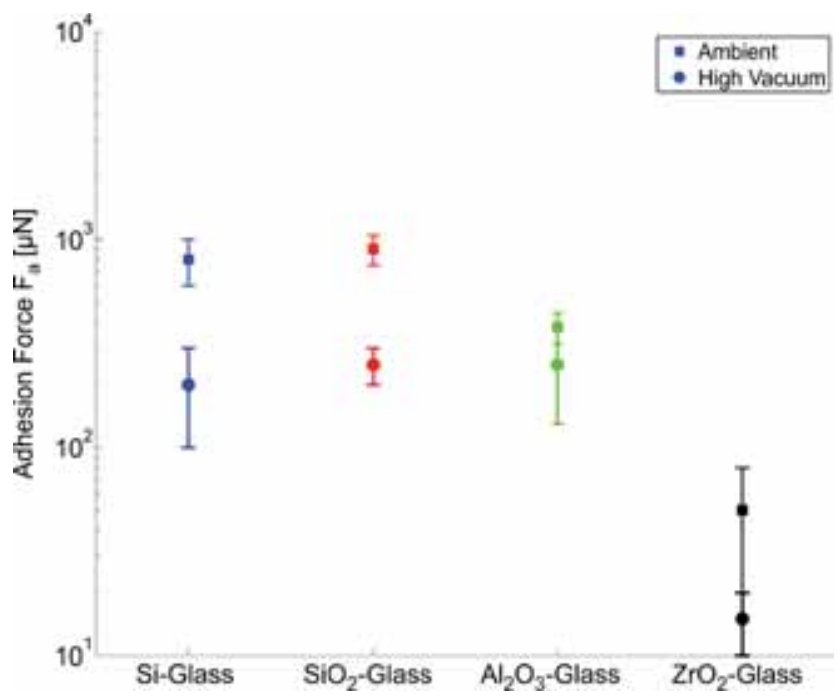

Figure 6: Adhesion force measurements for different material combinations in ambient as well as in HV conditions.

As explained earlier, this reduction in the adhesion force is strongly dependent on the radius of the sphere, contact area and roughness.

The results of adhesion measurements for different material combinations mentioned above are shown in Fig. 6. The measurements have been performed by applying a constant normal load of $10 \mathrm{mN}$ and by keeping a constant contact time of $5 \mathrm{sec}$ both in ambient and HV conditions. A significant difference can be seen when the measurements are performed in ambient and HV conditions. For all the material combinations, the adhesion force is reduced when measured in HV. On the other hand, a significant change in the adhesion force can be seen between relatively smooth $\mathrm{Si}-$ Glass and $\mathrm{SiO}_{2}$-Glass interfaces and relatively rough $\mathrm{Al}_{2} \mathrm{O}_{3}-$ Glass and rough $\mathrm{ZrO}_{2}-\mathrm{ZrO}_{2}$ interfaces. Each measurement point consists of at least 10 pull-off measurements, and the error bars show one standard deviation of the measurement data.

\subsection{Friction force measurements}

Experiments were performed to study the frictional behaviour of the Si-Glass interface. A typical force-displacement curve for measuring the friction force is shown in Fig. 7. The force measured with the force measuring mechanism in the tangential direction is plotted against the horizontal displacement of the $\mathrm{X}$ stage. These friction force loops show the first loading-unloading cycle in the tangential direction after the contact is made. The $\mathrm{X}$ stage moves towards right to load the contact tangentially as shown in Fig. 1a. In Fig. 7a, a friction loop is shown for measurements performed in ambient conditions. The flat surface is loaded with the desired load with the help of $\mathrm{Z}$ stage and then the tangential load is applied with the help of X stage. Similar friction force loops have been measured in high vacuum conditions and an example is shown in Fig. 7b. Moreover, the static and dynamic friction regimes are distinctively seen along with the preliminary displacement da and gross slip regions. In ambient conditions in this particular case the applied normal load is $6.5 \mathrm{mN}$, which resulted in 3.3 $\mathrm{mN}$ of friction force and $2 \mu \mathrm{m}$ of preliminary displacement. However, in the vacuum 


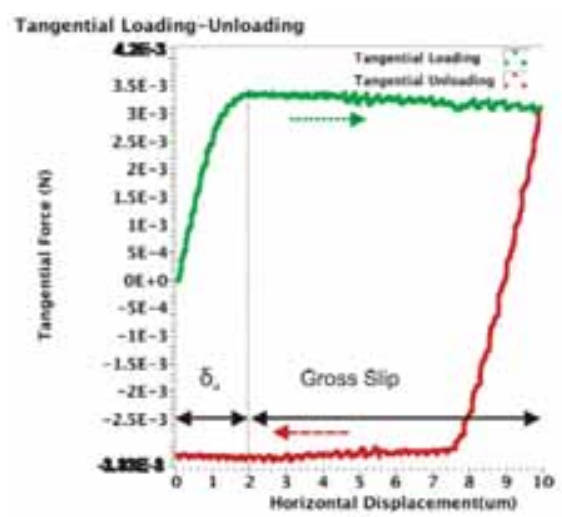

(a)

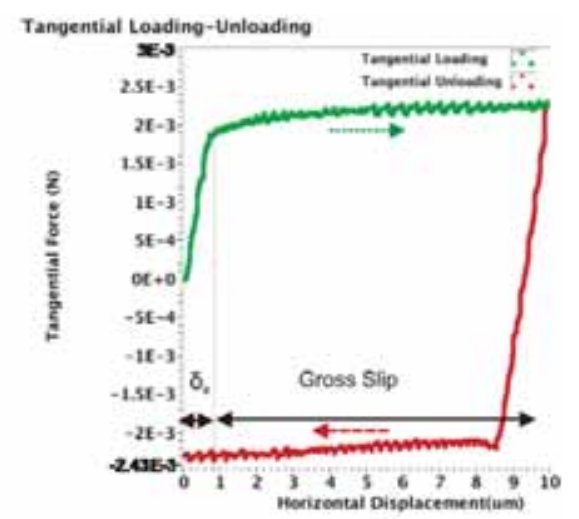

(b)

Figure 7: Typical friction force loops measuring friction force and preliminary displacement for Si-Glass interface in (a) ambient and (b) vacuum.

environment the applied normal load is $9 \mathrm{mN}$ and a friction force of $2 \mathrm{mN}$ with a preliminary displacement of $0.7 \mu \mathrm{m}$.

The coefficient of friction (COF) and preliminary displacement $\left(\delta_{\mathrm{a}}\right)$ as a function of applied normal load both in ambient and high vacuum conditions are shown in Fig. 8. A significant decrease is seen when the same system is operated in the high vacuum environment both for the preliminary displacement and the COF. According to Hertz theory, the contact area is proportional to the applied normal load to the power $2 / 3$ as shown in eqn (2) and the friction force is given as shown in eqn (3)

$$
\begin{gathered}
A=\pi\left(\frac{3}{4} \frac{R}{E^{*}}\right)^{2 / 3} F_{n}^{2 / 3}, \\
F_{t}=\tau A .
\end{gathered}
$$

The COF is defined by the following equation:

$$
\mu=\frac{F_{t}}{F_{n}} \Rightarrow \mu \propto \frac{F_{n}^{2 / 3}}{F_{n}}, \mu \propto F_{n}^{-1 / 3} .
$$

Assuming a constant shear stress $\tau$ of the interface, the COF is proportional to the applied normal load to the power $-1 / 3$ as shown in eqn (4). However, the Hertz theory does not incorporate adhesion effects that are important to consider at low applied normal loads as explained by Johnson [21]. JKR, DMT and M-D theories are few examples of incorporating adhesion effects. A comparison of contact area as a function of normal load for Hertz, JKR and DMT models is shown in Fig. 9 for Si ball of 5-mm diameter. The normal load dependency of the contact area as explained in eqn (2) by Hertz remains more or less same in JKR and DMT theories. Therefore, to extract the normal load dependency of the contact area even when the adhesion plays an important role Hertz theory can be used.

The curve fittings in Fig. 8a show that the measurements are in good agreement with the theory. Similarly, in Fig. $8 \mathrm{~b}$ the preliminary displacement $\delta_{\mathrm{a}}$ is plotted against the applied normal load. According to Mindlin [8] microslip occurs before gross slip when tangential load is applied to the contacting bodies. This microslip here is characterized as preliminary 


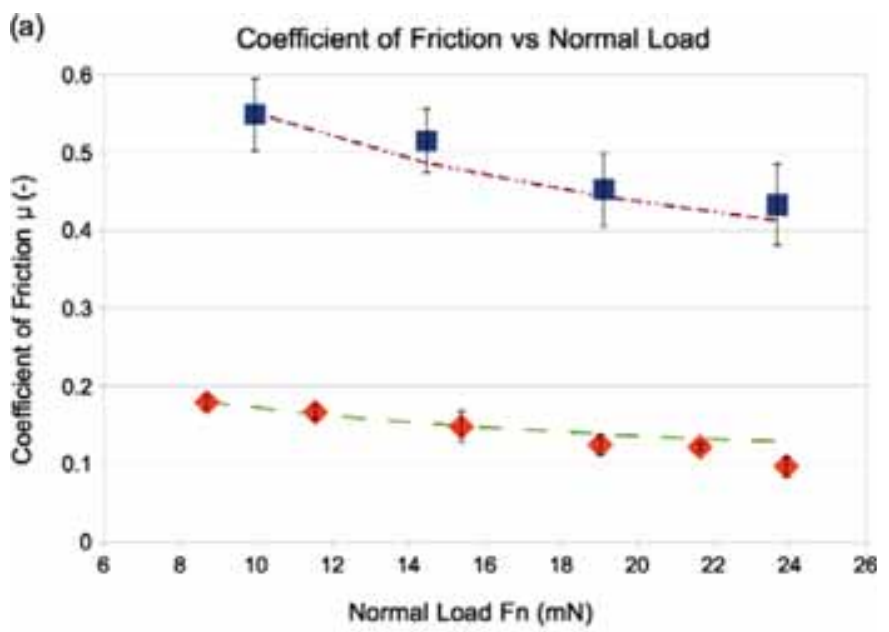

(b)

Preliminary Displacement vs Normal Load

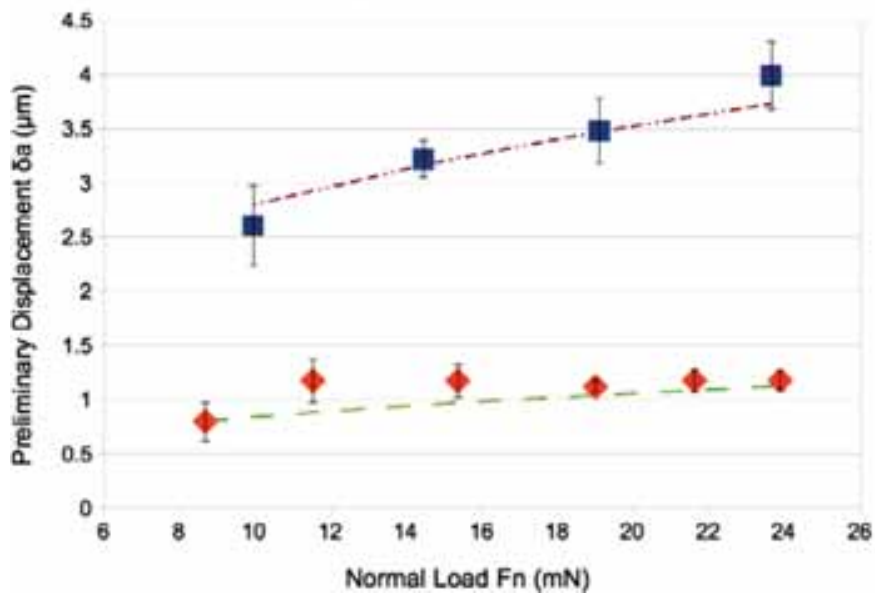

Figure 8: (a) Coefficient of friction and (b) preliminary displacement against normal load measured both in ambient $\mathbf{a n d}$ high vacuum $\mathrm{\text {for }} \mathrm{Si-Glass}$ system. Power fitting shows the validation of the results with theory.

displacement and is given by Galligan and McCullough [22]. The relationship between preliminary displacement $\delta_{\mathrm{a}}$ and applied normal load can be given as

$$
\delta_{a}=\frac{F_{t}}{8 a}\left(\frac{2-v_{1}}{G}\right) \Rightarrow \delta_{a} \propto \frac{\mu F_{n}}{a}, \quad \delta_{a} \propto \frac{F_{n}^{-1 / 3} F_{n}}{F_{n}^{1 / 3}}, \quad \delta_{a} \propto F_{n}^{1 / 3} .
$$

where $\delta_{\mathrm{a}}$ is directly proportional to the tangential load $\mathrm{Ft}$ and inversely proportional to the contact radius $\alpha$. The COF is proportional to the applied normal load to the power $-1 / 3$, and the contact radius is proportional to the applied normal load to the power $1 / 3$, this gives us the da proportional to the applied normal load to the power $1 / 3$ as shown in eqn (5). The experimental results shown in Fig. 8(b) are also in agreement with the theory since the increasing trend is following the $1 / 3$ power of normal load. 


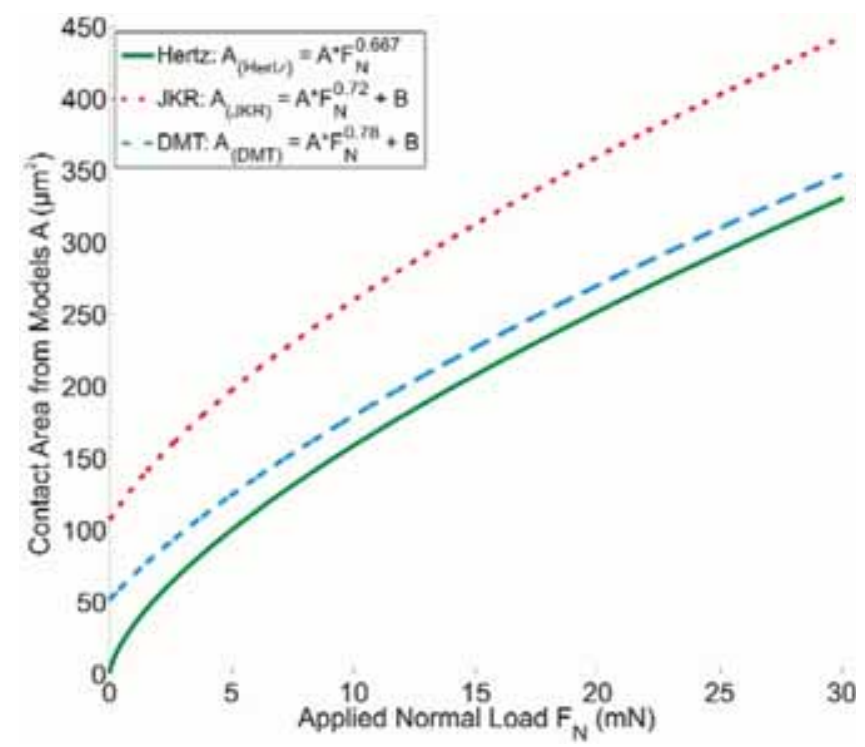

Figure 9: A comparison of the contact areas calculated using Hertz, JKR and DMT models for Si ball of 5-mm diameter against a glass flat surface.

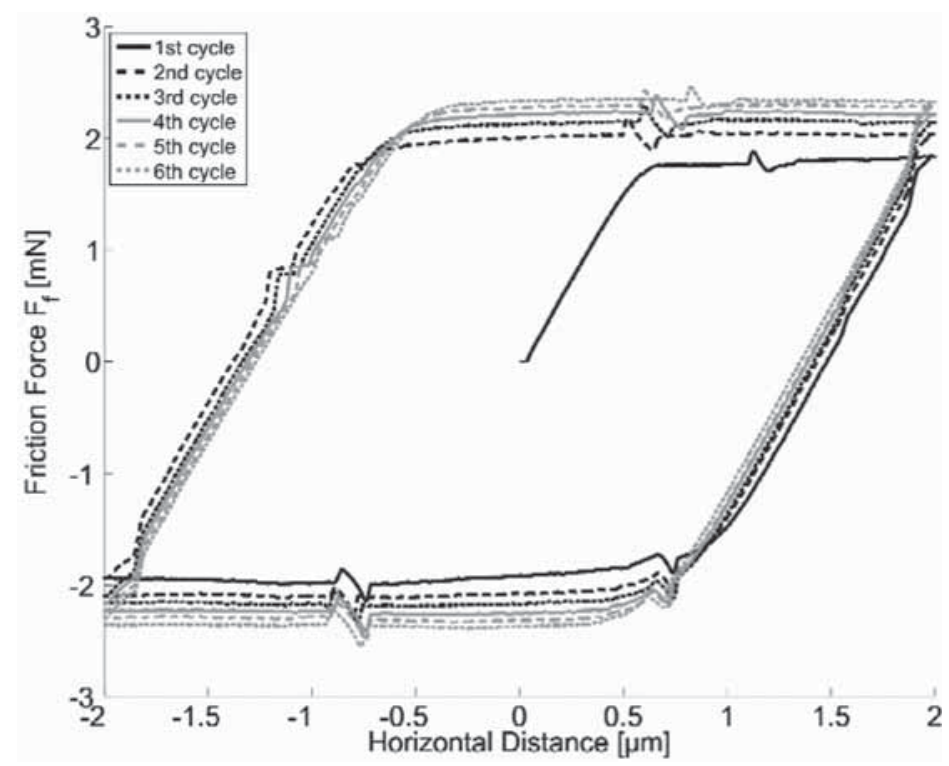

Figure 10: Friction force cycles measured with a 5- $\mathrm{mm} \mathrm{SiO}_{2}$ ball and a glass flat surface in ambient conditions with an applied normal load of $10 \mathrm{mN}$.

The friction force measurements were performed with a 5-mm diameter $\mathrm{SiO}_{2}$ ball and a glass flat surface in ambient conditions. The measurements were performed in a cyclic way to form friction loops as shown in Fig. 10. The normal load is applied using the $\mathrm{Z}$ stage and was kept $10 \mathrm{mN}$ for these measurements. Once the normal load is applied the tangential load is applied using the $X$ stage and is moved $2 \mu \mathrm{m}$ to the left. The stage is then moved $4 \mu \mathrm{m}$ to 
the right to complete one cycle. In Fig. 10, there are six cycles shown produced by reciprocating the tangential load. The X stage has been moved with a constant speed of $50 \mathrm{~nm} / \mathrm{sec}$ during this measurement. It can be seen that the friction force value for the first cycle is different than the measured friction force for other cycles. On the other hand, the friction force is increasing gradually with the increase in the no. of cycles. This can be explained by the presence of interfacial layers on the contacting surfaces. The first cycle removes most of the interfacial layer and causes a low value of friction force. In the second cycle, a significant increase in the friction force is seen and for the next cycles the increase in the friction force is not too significant. The distinctive static and dynamic friction regions can also be clearly seen.

\section{CONCLUSIONS}

A novel Vacuum Adhesion Friction Tester has been designed to perform adhesion and friction measurements both in ambient and vacuum conditions. The results show that the test rig is capable of measuring micro adhesion and friction. The setup is able to measure adhesion and friction force for both single asperity and multi-asperity contacts. The experimental results for the single asperity and multi-asperity contact show that the adhesion force reduces when the interface is operating in high vacuum. The adhesion force is strongly dependent on sphere radius, real contact area and the surface roughness of the interface. Measurements performed with different material combinations having different surface roughness and sphere radius show decrease in the adhesion force with the increase in surface roughness. Force-displacement curves for friction measurements for single asperity contact both in ambient and high vacuum conditions have been presented. Friction force is also reduced when the same system operates in high vacuum. The COF and the preliminary displacement also reduce for Si-Glass system in vacuum. The experimental results show good agreement with the basic theories of contact mechanics.

\section{ACKNOWLEDGEMENTS}

This research was carried out under project number MC7.06284 in the framework of the Research Program of the Materials innovation institute M2i (www.m2i.nl). Financial support for carrying out this research from the M2i is gratefully acknowledged.

\section{REFERENCES}

[1] Xiao, X. \& Qian, L., Investigation of humidity-dependent capillary force. Langmuir, 16, pp. 8153-8158, 2000. doi: http://dx.doi.org/10.1021/la000770o

[2] Israelachivili, J.N., Intermolecular and Surface Forces. 2nd ed, Elsevier Ltd: Amsterdam, 1991. doi: http://dx.doi.org/10.1116/1.1627336

[3] Bhushan, B., Adhesion and stiction: mechanisms, measurement techniques, and methods for reduction. Journal of Vacuum Science and Technology B, 21(6), pp. 2262-2292, 2003.

[4] van Zwol, P.J., Palasantzas, G., \& de Hosson, J.T.M., Influence of roughness on capillary forces between hydrophilic surfaces. Physical Review E, 78(031606), pp. 1-6, 2008.

[5] Johnson, K.L. \& Greenwood, J.A., An adhesion map for the contact of elastic spheres. Journal of Colloid and Interface Science, 192, pp. 326-333, 1997. doi: http://dx.doi. org/10.1006/jcis.1997.4984

[6] Feldman, K., Fritz, M., Hahner, G., Marti, A., \& Spencer, N.D., Surface forces, surface chemistry and tribology. Tribology International, 31(1-3), pp. 99-105, 1998. doi: http://dx.doi.org/10.1016/S0301-679X(98)00012-7 
[7] Ciavarella, M., The generalized Cattaneo partial slip plane contact problem. I - Theory. International Journal of Solids and Structures, 35(18), pp. 2349-2362, 1998. doi: http://dx.doi.org/10.1016/S0020-7683(97)00154-6

[8] Mindlin, R.D., Compliance of elastic bodies in contact. ASME Journal of Applied Mechanics. 16, pp. 259-268, 1949.

[9] Mindlin, R.D. \& Deresiewicz, H., Elastic spheres in contact under varying oblique forces. Journal of Applied Mechanics, 20, pp. 327-344, 1953.

[10] Amstrong-H'elouvry, B., Control of Machines With Friction, Kluwer Academic Publishers: Massachusetts and Dordrecht, 1991. doi: http://dx.doi.org/10.1007/978-1$\underline{4615-3972-8}$

[11] Capone, G., Della Valle, S., Guida, D. \& D'Agostino, V., On the friction-position control relationship. Proceedings of the Institution of Mechanical Engineers Part J: Journal of Engineering Tribology. 211(2), pp. 129-137, 1997. doi: http://dx.doi. org/10.1243/1350650971542372

[12] Yaqoob, M.A., de Rooij, M.B., \& Schipper, D.J., Design of a vacuum based test rig for measuring micro adhesion and friction force. High Performance Structures and Materials, VI, pp. 261-274, 2012.

[13] Awtar, S., Synthesis and analysis of parallel kinematic XY flexure mechanism, PhD Thesis, Massachusetts Institute of Technology, pp. 23-42, 2004.

[14] Xu, D., Liechti, K.M., \& Ravi-Chandar, K., On the modified tabor parameter for the JKR-DMT transition in the presence of a liquid meniscus. Journal of Colloid and Interface Science, 315, pp. 772-785, 2007. doi: http://dx.doi.org/10.1016/j.jcis.2007.07.048

[15] Harnett, E.M., Alderman, J. \& Wood, T., The surface energy of various biomaterials coated with adhesion molecules used in cell culture. Colloids and Interface Science B: Biointerfaces, 55, pp. 90-97, 2007. doi: http://dx.doi.org/10.1016/j.colsurfb.2006.11.021

[16] Krol, P. \& Krol, B., Determination of free surface energy values for ceramic materials and polyurethane surface-modifying aqueous emulsions. Journal of the European Ceramic Society, 26, pp. 2241-2248, 2006. doi: http://dx.doi.org/10.1016/j.jeurceramsoc. 2005.04.011

[17] Yoshizawa, H., Chen, Y.L. \& Israelachvili, J., Fundamental mechanisms of interfacial friction. Relation between adhesion and friction. Journal of Physical Chemistry, 97(16), pp. 4128-4140, 1993. doi: http://dx.doi.org/10.1021/j100118a033

[18] Liu, L. \& Bushan, B., Adhesion and friction studies of microelectromechanical systems/nanoelectromechanical systems materials using a novel microtriboapparatus. Journal of Vacuum Science and Technology A, 21(4), pp. 1528-1538, 2003. doi: http:// dx.doi.org/10.1116/1.1560711

[19] Grobelny, J., Pradeep, N., Kim, D.I. \& Ying, Z.C., Quantification of the meniscus effect in adhesion force measurement. Applied Physics Letters, 88(091906), pp. 091906-1091906-3, 2006. doi: http://dx.doi.org/10.1063/1.2181200

[20] Rabinovich, Y.I., Alder, J.J., Ata, A., Singh, R.K. \& Moudgil, B.M., Adhesion between nanoscale rough surfaces II. Measurement and comparison with theory. Journal of Colloid and Interface Science, 232, pp. 17-24, 2000. doi: http://dx.doi.org/10.1006/jcis. 2000.7168

[21] Johnson, K.L., Mechanics of adhesion. Tribology International, 31(8), pp. 413-418, 1998. doi: http://dx.doi.org/10.1016/S0301-679X(98)00060-7

[22] Galligan, J.M. \& McCullough P., On the nature of static friction. Wear, 105, pp. 337-340, 1985. doi: http://dx.doi.org/10.1016/0043-1648(85)90232-7 